\title{
CONCENTRACIONES DE ARSÉNICO URINARIO EN POBLADORES DE DOS DISTRITOS DE LA REGIÓN TACNA, PERÚ, 2017
}

\author{
Diego A. Ale-Mauricio ${ }^{1, a}$, Guillermo Villaª,a, María del Carmen Gastañaga ${ }^{2, b}$
}

\begin{abstract}
RESUMEN
Objetivos. Determinar la concentración de arsénico (As) total urinario en pobladores adultos de dos distritos de la región Tacna. Materiales y métodos. Estudio transversal realizado en los distritos de Cairani y Camilaca, en la provincia de Candarave en la región Tacna, Perú, para lo cual se recolectaron 103 y 71 muestras de orina, respectivamente. La concentración de As urinario se determinó por el método de digestión en microondas e inyección de flujo en espectrofotometría de absorción atómica. Resultados. La mediana de concentración de As urinario en Cairani fue de $601,6 \mu \mathrm{g} / \mathrm{g}$ creatinina (RIC 407,3 - 847,1) y en Camilaca fue de 30,2 $\mu \mathrm{g} / \mathrm{g}$ creatinina (RIC 21,4 - 39,7). El 100 \% (103) de las muestras procedentes de Cairani y el 80,3 \% (57) de las muestras de Camilaca superaron los valores referenciales de toxicidad. Asimismo, los pobladores de Cairani superan en 30 veces, y los de Camilaca en 1,5 veces, los valores de toxicidad para As. Conclusiones. La población adulta de los distritos de Cairani y Camilaca de la provincia de Candarave, de la región Tacna, tienen concentraciones de As urinario superiores a los valores referenciales de toxicidad establecidos por la Organización Mundial de la Salud.
\end{abstract}

Palabras clave: Arsénico; Agua subterránea; Valores de referencia; Perú (fuente: DeCS BIREME).

\section{URINARY ARSENIC CONCENTRATIONS IN ADULT POPULATION OF TWO DISTRICTS FROM THE TACNA REGION, PERU, 2017}

\begin{abstract}
Objectives. To determine the concentration of total urinary arsenic (As) in the adult population of two districts in the Tacna region. Materials and Methods. A cross-sectional observational study conducted in the districts of Cairani and Camilaca, in the province of Candarave in the Tacna region, Peru. For the study 103 and 71 urine samples were collected. The concentration of urinary As was determined by the method of microwave digestion and flow injection in atomic absorption spectrophotometry (FIAS-AAS). Results. In the Cairani district, a median urinary arsenic concentration of $601.61 \mu \mathrm{g} / \mathrm{g}$ creatinine (IQR: 407,3 - 847,1) was obtained; and for Camilaca, a median of $30.24 \mu \mathrm{g} / \mathrm{g}$ creatinine was obtained (IQR: 21,4 - 39,7). The residents of Cairani and Camilaca exceed 30 and 1.5 times, respectively, the toxicity reference values (TRV) for arsenic. Conclusions. The adult population of the districts of Cairani and Camilaca in the province of Candarave of the Tacna region has urinary As concentrations exceeding the toxicity reference values (TRV) established by the World Health Organization (WHO).
\end{abstract}

Keywords: Arsenic; Groundwater; Reference values; Peru (source: MeSH NLM).

\section{INTRODUCCIÓN}

Actualmente, la contaminación ambiental, es uno de los problemas de salud más críticos que afrontan los países en desarrollo en todo el mundo. Entre los principales agentes contaminantes está el arsénico (As), el cual es uno de los 20 elementos más comunes de la corteza terrestre y uno de los 14 elementos traza del agua de mar. Además, es considerado un factor de riesgo para la salud global debido a su carácter tóxico. La capacidad geoquímica del As permite su filtración, de menor a mayor concentración, en el agua de consumo humano a través de procesos naturales de interacción agua roca, ocasionada por ambientes geológicos específicos ${ }^{(1,2)}$. Por ello, la Organización

\footnotetext{
Facultad de Ciencias de la Salud, Universidad Nacional Jorge Basadre Grohmann. Tacna, Perú.

Centro Nacional de Salud Ocupacional y Protección del Ambiente para la Salud. Instituto Nacional de Salud. Lima, Perú.

Químico farmacéutico; ${ }^{\mathrm{b}}$ médico

Recibido: 23/05/2018 Aprobado: 27/06/2018 En línea: 06/07/2018
}

Citar como: Ale-Mauricio DA, Villa G, Gastañaga MC. Concentraciones de arsénico urinario en pobladores de dos distritos de la región Tacna, Perú, 2017. Rev Peru Med Exp Salud Publica. 2018;35(2):183-9. doi: 10.17843/rpmesp.2018.352.3693. 
Mundial de la Salud (OMS) ha establecido el valor de 10 $\mu \mathrm{g} / \mathrm{L}$ como límite de concentración de As en el agua potable para consumo humano ${ }^{(3)}$.

En la región Tacna, en Perú, la presencia de As en el agua de consumo humano es un problema condicionado esencialmente por su ubicación geográfica, en suelos volcánicos que generan la contaminación natural de aguas geogénicas por la disolución del As ${ }^{(4)}$. Un informe institucional realizado por la Dirección Regional de Salud de Tacna en 2014 reportó que 19 de 27 distritos (70 \%) exceden los límites máximos permisibles de As en agua de consumo humano establecido por la OMS y la legislación peruana ${ }^{(5,6)}$. Asimismo, un estudio ejecutado en 2015 por la Dirección de Salud Ambiental de Tacna reportó concentraciones de $270 \mu \mathrm{g} / \mathrm{L}$ a $680 \mu \mathrm{g} / \mathrm{L}$ de As en aguas subterráneas del distrito de Cairani en la provincia de Candarave ${ }^{(7)}$.

Diversos estudios reportan la asociación entre la ingesta crónica de agua con As y la presencia de enfermedades crónicas como la diabetes mellitus, hipertensión arterial, anemia megaloblástica, trastornos gastrointestinales y lesiones arsenicales en la piel (melanosis, melanodermia, banda de Mees, discromía en formas de gotas de lluvia y queratosis) ${ }^{(8-10)}$.

La Agencia para Sustancias Tóxicas y el Registro de Enfermedades (ASTDR) ${ }^{(11)}$ y el Ministerio de Salud (8) sugieren que la exposición crónica se determine mediante la cuantificación del As total en orina, estableciendo un valor referencial de toxicidad de $20 \mu \mathrm{g} / \mathrm{g}$ creatinina ${ }^{(12)}$.

Apesar de los informes técnicos que evidencian la exposición crónica a As en la población de Candarave, se desconoce la concentración del tóxico en la población residente. El presente estudio busca determinar la concentración de As total urinario en los pobladores adultos de los distritos de Cairani y Camilaca, cuyas aguas subterráneas presentan contaminación natural con As.

\section{MATERIALES Y MÉTODOS}

\section{DISEÑO Y ÁMBITO DE ESTUDIO}

Se realizó un estudio transversal en los distritos de Cairani y Camilaca en la provincia de Candarave, región Tacna. Los distritos estudiados se ubican entre los 3400 y 3850 metros sobre el nivel del mar. La Dirección Ejecutiva de Salud Ambiental de Tacna indica que la fuente de agua de consumo de ambos distritos es de origen subterránea, recibiendo aportes del río Callazas (Cairani) y del manantial Japo (Camilaca) (Figura 1). Por otro lado, se reporta que el agua que consumen los pobladores de los distritos de Cairani y Camilaca presentan concentraciones de As de $680 \mu \mathrm{g} / \mathrm{L}$ y $2 \mu \mathrm{g} / \mathrm{L}$, respectivamente ${ }^{(4,13)}$.

\section{MENSAJES CLAVE}

Motivación para realizar el estudio. En los distritos de Cairani y Camilaca en la región Tacna, la presencia de arsénico (As) en el agua de consumo es un problema plenamente identificado.

Principales hallazgos. La mediana de As urinario en Cairani fue de $601,6 \mu \mathrm{g} / \mathrm{g}$ creatinina y en Camilaca fue de $30,2 \mu \mathrm{g} / \mathrm{g}$ creatinina, estos valores superan en 30 veces y en 1,5 veces, los valores referenciales de toxicidad para As.

Implicancias. Se debe propiciar el seguimiento a la salud de los pobladores Cairani y Camilaca; asimismo, se debe de promover la aplicación de tecnologías que contribuyan a la reducción de las concentraciones de As en agua de consumo.

\section{POBLACIÓN DE ESTUDIO}

Según los datos proporcionados por la Dirección Regional de Salud de Tacna, para el 2016 la cantidad de residentes de Cairani y Camilaca fue de 607 y 670 habitantes, respectivamente ${ }^{(14)}$.

Considerando las cantidades poblacionales, se realizó el cálculo del tamaño de ambas muestras, con un nivel de confianza de $95 \%$, un margen de error relativo del 0,07, una proporción esperada del $50 \%$, y un tamaño de estrato de 0,52 para Cairani y 0,47 para Camilaca. Finalmente, el tamaño muestral calculado para Cairani fue de 103 pobladores y para Camilaca fue de 71 pobladores. Asimismo, se realizó un muestreo aleatorio estratificado con afijación proporcional al tamaño. El estudio fue ejecutado entre los meses de enero y febrero del 2017.

Se incluyeron a pobladores mayores de 18 años, con un tiempo mínimo de residencia de 12 meses, y que hayan brindado su consentimiento informado. Previamente a la selección de los participantes, se aplicó una encuesta personal estructurada, así como la verificación de la historia clínica de cada aspirante. Se excluyeron a pobladores alcohólicos, fumadores, con enfermedades agudas o crónicas del tracto urinario, expuestos ocupacionalmente (trabajadores mineros, operadores de fundición, aplicadores de pesticidas/insecticidas) y pobladores que hayan consumido pescado o mariscos dentro de las 48 horas previas a la toma de muestra ${ }^{(11,15)}$.

\section{VARIABLES DE ESTUDIO}

Las variables sociodemográficas consideradas en el estudio fueron sexo, edad, distrito de procedencia, tiempo de residencia y ocupación. La variable edad se identificó como numérica continua y para la expresión de resultados se mostró en rangos de quindenios a partir de los 20 años. Para la obtención de la información de cada variable se utilizó con una ficha de recolección de datos. La variable de interés fue la exposición al As medido a través de su concentración en orina, se consideró como punto de corte el valor de referencia establecido (20 $\mu \mathrm{g} / \mathrm{g}$ creatinina). 


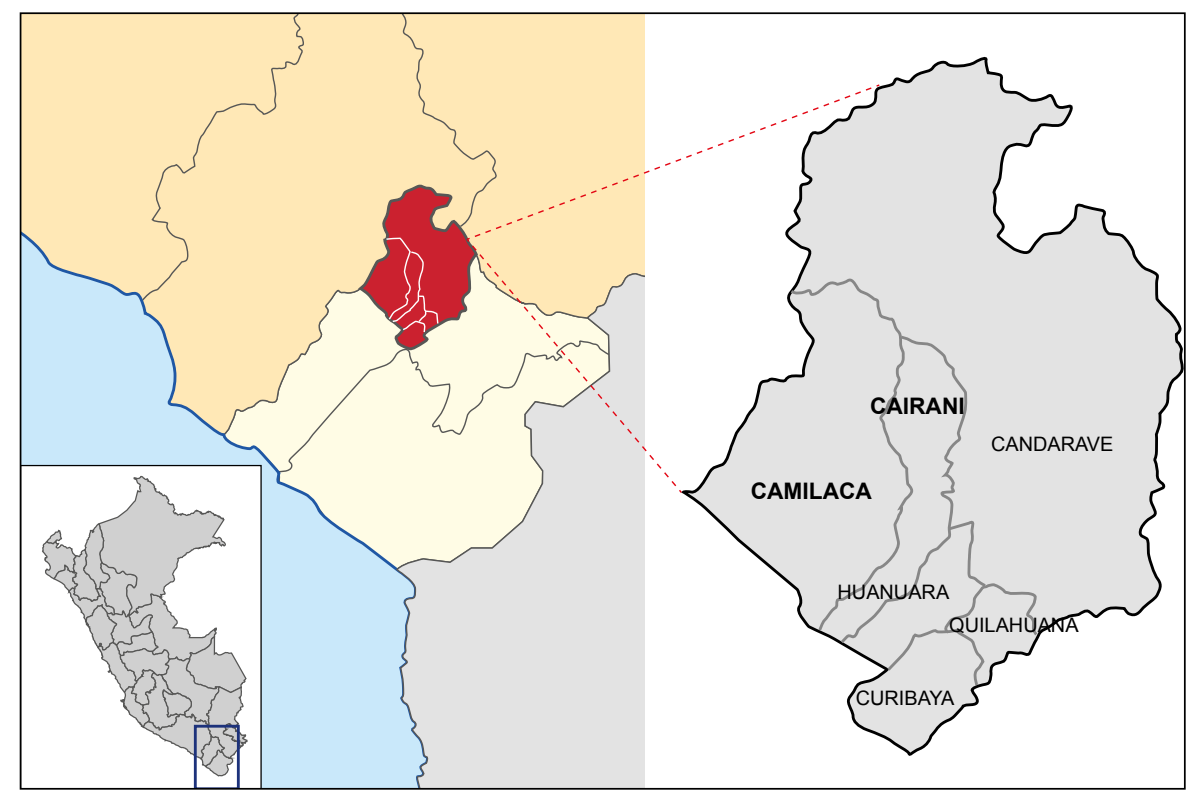

Figura 1. Ubicación geográfica de los distritos de Cairani y Camilaca en la provincia de Candarave, región Tacna

\section{PROCEDIMIENTOS E INSTRUMENTOS}

La recolección de muestras de orina se realizó de acuerdo con el procedimiento técnico del Centro Nacional de Salud Ocupacional y Protección del Ambiente para la Salud (CENSOPAS), que señala las pautas de colección, conservación, almacenamiento, transporte y recepción de muestras biológicas para la determinación de metales pesados ${ }^{(16)}$.

Se realizaron reuniones con la población, con el personal médico de los puestos de salud y con las autoridades municipales de ambos distritos. El personal médico revisó las historias clínicas de los voluntarios. Finalmente, considerando los criterios de inclusión se seleccionaron a los participantes.

La toma de muestra fue programada en un día de trabajo de campo, citando a los pobladores durante la mañana, a fin de recolectar la primera orina del día. Para la obtención de las muestras se acondicionó dos áreas en el puesto de salud de cada distrito, que tuvieran las siguientes condiciones: ambiente cerrado, temperatura y humedad adecuada, sin emanaciones de polvo y con buena iluminación. En la primera área, previamente limpiada y aséptica, se colectó la muestra de orina; en la segunda área, el ambiente fue acondicionado para el tratamiento de la muestra. Se le entregó a cada participante una hoja con instrucciones para la colección de la muestra. Adicionalmente se le proporcionó un frasco estéril de $100 \mathrm{ml}$ con tapa rosca, una chaqueta estéril y descartable y guantes de nitrilo. Posteriormente se recepcionó la muestra, se verificaron los datos del participante y se le asignó un identificador único. Las muestras se conservaron en cadena de frío entre $4{ }^{\circ} \mathrm{C}$ a $8^{\circ} \mathrm{C}$.

Las muestras fueron analizadas y evaluadas en el laboratorio químico toxicológico y en el laboratorio clínico del CENSOPAS del Instituto Nacional de Salud. El ensayo para cuantificar As en muestras de orina fue realizado por espectroscopia de absorción atómica con generación de hidruros asistido por microondas, metodología validada según un procedimiento técnico establecido. El método de análisis cuenta con controles internos y externos de calidad.

Para el control interno se emplearon materiales de referencia certificados; tales como BIORAD®, CLINCHECK® y NIST 2670a; mientras que, el control externo de calidad, se realizó a través de un convenio con el Instituto Nacional de Salud Pública de Quebec en Canadá. Asimismo, el laboratorio de referencia cuenta con un programa de aseguramiento de la calidad, basado en los requisitos de la norma técnica ISO 15189.

Se utilizó un equipo de microondas con capacidad hasta de $240^{\circ} \mathrm{C}$, un equipo de absorción atómica con AutoSampler y un equipo de inyección de flujo. El rango de trabajo estuvo entre $1 \mu \mathrm{g} / \mathrm{L}$ a $20 \mu \mathrm{g} / \mathrm{L}$ de As en orina, además de un límite de detección de $5 \mu \mathrm{g} / \mathrm{L}$. El método tiene una precisión menor al $20 \%$ respecto a la reproducibilidad.

A cada muestra de orina se adicionó persulfato de potasio al $3 \%$ obteniendo diluciones de 1:10, luego se digestó en el equipo de microondas por 90 minutos a $200{ }^{\circ} \mathrm{C}$ con el objetivo de oxidar todos los compuestos químicos del $\mathrm{As}^{+3}$ (trivalente) a $\mathrm{As}^{+5}$ (pentavalente). 
Para el control de precisión del ensayo, todas las muestras fueron digestadas por duplicado considerando que la desviación estándar relativa sea menor a $20 \%$.

Luego de la digestión se realizó una reducción con ácido ascórbico al $5 \%$ y yoduro de potasio al $5 \%$ (solución reductora). A cada muestra digestada se le realizó diluciones de 2:15. Posteriormente se añadió $5 \mathrm{ml}$ de ácido clorhídrico concentrado a cada muestra y se dejó reposar por 45 minutos. Por último, las muestras se colocaron en el equipo de inyección de flujo donde se formaron hidruros volátiles en medio ácido por adición de borohidruro de sodio. El hidruro volátil fue arrastrado por una corriente de gas argón y atomizado en una cámara de cuarzo previamente calentada y colocada en el camino óptico de la radiación que absorbe el As a una longitud de onda 193,7 $\mathrm{nm}$. La cuantificación se efectuó por interpolación en una curva de calibración.

Por otra parte, la determinación de creatinina en orina, se realizó por el método colorimétrico de reacción de Jaffé con espectrofotómetro ultravioleta.

\section{ANÁLISIS ESTADÍSTICO}

Se empleó estadística descriptiva (medianas y rangos intercuartílicos), además de estadística inferencial bivariada no paramétrica considerando la mediana para el análisis y utilizando la prueba de $U$ de Mann Whitney y ANOVA de Kruskall Wallis, cuando correspondía. Previamente se evaluó la normalidad de los datos con la prueba de Kolmogorov-Smirnov con corrección de Lilliefors. Se consideró un valor de $p<0,05$ como estadísticamente significativo. El procesamiento de datos se realizó en los programas Microsoft Excel y SPSS versión 24.

\section{CONSIDERACIONES ÉTICAS}

El estudio fue aprobado por el Comité de Ética en Investigación del Instituto Nacional de Salud. Los pobladores fueron invitados a participar de manera voluntaria, se les explicó los objetivos del estudio y firmaron un consentimiento informado. Los resultados se entregaron a la Dirección Regional de Salud de Tacna, quienes se encargaron de su distribución a los establecimientos de salud de cada distrito, para que sean entregados a todos los participantes. La entrega fue de manera individual, a cargo de un equipo médico. Asimismo, los resultados se adjuntaron en las historias clínicas de los participantes.

\section{RESULTADOS}

Los participantes del distrito de Cairani presentaron una mediana de $601,6 \mu \mathrm{g} / \mathrm{g}$ creatinina (RIC 407,3 - 847,1) y los de
Camilaca presentaron una mediana de $30,2 \mu \mathrm{g} / \mathrm{g}$ creatinina (RIC 21,4 - 39,7), siendo diferentes estadísticamente $(p<0,001)$. La mediana de As en los pobladores de Cairani fue 19,9 veces superior a la mediana de los pobladores de Camilaca. Los valores máximos de concentración de As urinario fueron de $1691,9 \mu \mathrm{g} / \mathrm{g}$ creatinina en Cairani y de $121,7 \mu \mathrm{g} / \mathrm{g}$ creatinina en Camilaca.

Las frecuencias de las características sociodemográficas de los pobladores de los distritos de Cairani y Camilaca se detallan en la Tabla 1.

Respecto a la proporción de pobladores que presentaron concentraciones tóxicas de As en orina, se obtuvo que $100 \%$ (103) de las muestras procedentes de Cairani y el $80,3 \%$ (57) de las muestras de Camilaca superaron en 30 y 1,5 veces, respectivamente, los valores referenciales de toxicidad (As $\geq 20 \mu \mathrm{g} / \mathrm{g}$ creatinina).

Respecto al sexo, las mujeres de Cairani presentan una mediana de As urinario (633,6; RIC 408,5 - 859,2) superior a los hombres, mientras que en Camilaca son los hombres quienes presentan una mayor mediana de As $(32,7$; RIC 26,6 - 46,1). Asimismo, los ganaderos de Cairani y los agricultores de Camilaca son los que tienen la mayor mediana de As urinario (973,7; RIC 740,9 - 1213,0 y 31,7; RIC 26,1 - 42,9 $\mu \mathrm{g} / \mathrm{g}$ creatinina, respectivamente), en comparación con otras ocupaciones (Tabla 2).

Tabla 1. Características sociodemográficas de los pobladores de los distritos de Cairani y Camilaca. Tacna, Perú 2017

\begin{tabular}{lcc}
\hline $\begin{array}{l}\text { Características } \\
\text { sociodemográficas }\end{array}$ & $\begin{array}{c}\text { Cairani } \\
\mathbf{n = 1 0 3}\end{array}$ & $\begin{array}{c}\text { Camilaca } \\
\mathbf{n = 7 1}\end{array}$ \\
\hline $\begin{array}{l}\text { Sexo (\%) } \\
\text { Masculino }\end{array}$ & $48(46,6)$ & $27(38,0)$ \\
\hline Femenino & $55(53,4)$ & $44(62,0)$ \\
\hline Edad (años) & $28(27,2)$ & $14(19,7)$ \\
\hline 20 a 35 & $24(23,3)$ & $22(31,0)$ \\
\hline 6 a 51 & $26(25,2)$ & $22(31,0)$ \\
\hline 52 a 67 & $25(24,3)$ & $13(18,3)$ \\
\hline 68 a 83 & $16(15,5)$ & $11(15,5)$ \\
\hline Tiempo de residencia (años) & $26(25,2)$ & $15(21,1)$ \\
\hline 1 a 15 & $17(16,5)$ & $19(26,8)$ \\
16 a 31 & $18(17,5)$ & $18(25,4)$ \\
\hline 32 a 47 & $26(25,2)$ & $8(11,3)$ \\
48 a 63 & & \\
\hline 64 a 83 & $82(79,6)$ & $43(60,6)$ \\
\hline Ocupación (\%) & $3(2,9)$ & $17(23,9)$ \\
\hline Agricultor & $10(9,7)$ & $11(15,5)$ \\
\hline Comerciante & $4(3,9)$ & - \\
\hline Ama de casa & $3(2,9)$ & - \\
\hline Ganadero & $1(1,0)$ & - \\
\hline Transportista & & \\
\hline Otro & & \\
\hline
\end{tabular}




\section{DISCUSIÓN}

Los pobladores de los distritos de Cairani y Camilaca tienen concentraciones de As total en orina de 84,6 y 6,1 veces más alto que el valor referencial de toxicidad considerado en la normativa nacional ${ }^{(8)}$.

El $100 \%$ de los pobladores de Cairani presenta concentraciones tóxicas de As en orina, con un valor máximo de $1691,89 \mu \mathrm{g} / \mathrm{g}$ creatinina. Estos resultados se asemejan a lo reportado por Chakraborti et al. ${ }^{(17)}$ en la India, donde los pobladores expuestos a fuentes de agua subterránea tienen un promedio de As en orina de $978 \mu \mathrm{g} / \mathrm{g}$ creatinina con un valor máximo de $1442 \mu \mathrm{g} / \mathrm{g}$ creatinina. Además, los autores reportan la presencia de lesiones dérmicas arsenicales en los pobladores expuestos.

Diversas investigaciones indican que las poblaciones expuestas crónicamente al As a través del agua de consumo tienen aproximadamente un riesgo de cáncer entre 30 a 300 veces más alto que el originado por cualquier otro cancerígeno ${ }^{(18)}$. Además de esta evidencia, otros estudios reportan la asociación entre la exposición crónica de As a través de la ingesta de agua y la presencia de enfermedades crónicas como diabetes, hipertensión arterial, anemia megaloblástica, trastornos gastrointestinales y lesiones arsenicales en la piel (melanosis, melanodermia, líneas de Mees, discromía en formas de gotas de lluvia y queratosis) ${ }^{(9,10)}$.
Otros estudios refieren concentraciones inferiores de As urinario; Hurtado ${ }^{(19)}$, reportó una concentración promedio de $47,6 \mu \mathrm{g} / \mathrm{g}$ creatinina, 14 veces menor que nuestros resultados, en pobladores del distrito de Sama en Tacna. Asimismo, Pérez Vásquez ${ }^{(20)}$ reportó una concentración promedio de $36,5 \mu \mathrm{g} / \mathrm{g}$ creatinina, 16,5 veces menor que la mediana encontrada en Cairani, en el distrito de Ite en Tacna. Es importante señalar que, en ambos estudios, la fuente de exposición fue el agua de origen superficial (ríos), el cual tiene compuestos orgánicos de As con menor grado de toxicidad, en comparación con el agua de origen subterráneo que tiene una alta concentración de $\mathrm{As}^{+3}$ (inorgánico) ${ }^{(21)}$.

Por otro lado, en Camilaca el 80,3 \% de la población adulta supera el valor referencial de As urinario. Asimismo, la mediana obtenida de $30,2 \mu \mathrm{g} / \mathrm{g}$ creatinina supera sólo en 1,5 veces el valor referencial de toxicidad. A pesar de que los niveles de As en el agua de consumo de Camilaca son inferiores a los límites máximos permisibles, este tiende a acumularse progresivamente, en la piel, cabello y uñas, por lo que este As acumulado se excretaría primordialmente por la orina en un $70 \%$ (22).

Peña et al. (23) indican que algunos tóxicos presentan respuestas diferentes dependiendo del sexo del organismo expuesto. Algunas de estas desigualdades se pueden explicar por las diferencias hormonales (testosterona o estrógeno) que afectan los procesos de absorción,

Tabla 2. Concentración de arsénico en orina según características sociodemográficas en pobladores de los distritos de Cairani y Camilaca. Tacna, Perú 2017

\begin{tabular}{|c|c|c|c|c|}
\hline \multirow{2}{*}{$\begin{array}{l}\text { Características } \\
\text { sociodemográficas }\end{array}$} & \multicolumn{2}{|c|}{ Cairani } & \multicolumn{2}{|c|}{ Camilaca } \\
\hline & Mediana (RIC) & Valor de $p^{*}$ & Mediana (RIC) & Valor de $\mathrm{p}^{\star}$ \\
\hline Sexo & & 0,341 & & 0,035 \\
\hline Masculino & $571,4(382,8-839,3)$ & & $32,7(26,6-46,1)$ & \\
\hline Femenino & $633,6(408,5-859,2)$ & & $27,7(19,6-38,3)$ & \\
\hline Edad (años) & & 0,130 & & 0,630 \\
\hline 20 a 35 & $555,4(392,1-684,1)$ & & $27,9(23,8-39,3)$ & \\
\hline 36 a 51 & $645,7(376,8-995,1)$ & & $33,1(26,9-37,1)$ & \\
\hline 52 a 67 & $713,2(515,7-942,9)$ & & $28,6(21,1-40,5)$ & \\
\hline 68 a 83 & $539,8(376,4-692,7)$ & & $23,6(10,6-48,6)$ & \\
\hline Tiempo de residencia (años) & & 0,989 & & 0,556 \\
\hline 1 a 15 & $594,7(397,1-925,2)$ & & $28,9(23,6-38,8)$ & \\
\hline 16 a 31 & $614,6(426,3-774,9)$ & & $26,8(23,3-36,7)$ & \\
\hline 32 a 47 & $505,8(370,9-959,6)$ & & $32,6(24,0-42,9)$ & \\
\hline 48 a 63 & $644,6(400,4-864,6)$ & & $29,4(19,2-36,4)$ & \\
\hline 64 a 83 & $596,8(399,2-798,6)$ & & $41,1(20,1-70,3)$ & \\
\hline Ocupación & & 0,076 & & 0,188 \\
\hline Agricultor & $581,5(392,4-788,8)$ & & $31,7(26,1-42,9)$ & \\
\hline Comerciante & $777,7(588,8-941,1)$ & & $29,6(21,1-36,0)$ & \\
\hline Ama de casa & $496,7(407,3-768,1)$ & & $23,6(12,3-39,7)$ & \\
\hline Ganadero & $973,7(740,9-1213,0)$ & & - & \\
\hline
\end{tabular}

RIC: Rango intercuartílico.

* Prueba U de Mann Whitney para variables con dos categorías y ANOVA de Kruskall Wallis para variables con más de dos categorías. 
distribución, biotransformación y excreción. Sin embargo, se debe considerar otros factores asociados a la distribución y excreción de As, tales como; la composición proteica, el estado nutricional y el tiempo de exposición al tóxico.

Freeman ${ }^{(24)}$ indica que el consumo de folatos y cisteína en la dieta contribuye a la metilación y eliminación del As. La composición proteica del individuo sirve como sustrato en la distribución del As, este tiende a enlazarse a proteínas unidas al zinc y a grupos sulfhidrilos, lo que ocasiona que se aglomere en los tejidos y, por ende, disminuya su concentración en orina. Esta podría ser una razón por la que los hombres de Cairani tienen concentraciones inferiores de As en orina, comparado con las mujeres.

Asimismo, en Camilaca la mayoría de las mujeres laboran en comercio y la totalidad de hombres laboran en agricultura, por lo que estos últimos estarían más expuestos al As que sus pares femeninos. Abernathy et al. (21) reportó que, a mayor tiempo de exposición, el hígado pierde su capacidad de metilar y, por lo tanto, se produce una mayor excreción de As inorgánico por la orina. Esto podría explicar la mayor mediana de As urinario en los hombres de Camilaca, en comparación con las mujeres.

Los resultados de este estudio conllevan a poner mayor atención y seguimiento a la salud de los pobladores Cairani y Camilaca debido a que la exposición al As, a través del agua de consumo, estaría asociado a cuadros de hidroarsenicismo crónico regional endémico (HACRE) ${ }^{(12)}$. Se debe tener en cuenta la aplicación de nuevas tecnologías que contribuyan en la reducción de las concentraciones de
As en el agua de consumo y por consiguiente preservar la salud de las poblaciones expuestas.

Se debe reconocer que la presente investigación limita sus resultados sólo a los distritos en estudio, y no a otros distritos de la provincia de Candarave. Se recomienda aumentar el tamaño muestral y realizar investigaciones en otros distritos y provincias de la región Tacna.

En conclusión, la población adulta de los distritos de Cairani y Camilaca de la provincia Candarave, en la ciudad de Tacna, tienen concentraciones tóxicas de As urinario, superiores a los valores referenciales establecidos por la OMS.

Agradecimientos: Al equipo técnico y profesional de la Dirección Regional de Salud Tacna quienes brindaron las facilidades de planificación y ejecución del estudio a través del Comité de Metales Pesados, al Dr. Claudio Ramírez, a la Lic. Blanca Rosado, a la Blga. Verónica Machaca y a la Blga. Juana Sosa. Al equipo profesional del Centro Nacional de Salud Ocupacional y Protección del Ambiente para la Salud, al Q.F. Arturo Erazo y al toxicólogo José Huamaní por su apoyo y participación en el estudio.

Contribuciones de autoría: DAAM y GV han participado en la concepción y diseño del artículo además de la recolección de datos y la redacción. DAAM realizó el análisis e interpretación de datos. MCG realizó la revisión crítica del artículo y aprobación de la versión final.

Fuente de Financiamiento: Instituto Nacional de Salud mediante Resolución Directoral N871-2016-OGITT-OPE/INS.

Conflictos de Interés: No.

\section{REFERENCIAS BIBLIOGRÁFICAS}

1. Mandal BK, Suzuki KT. Arsenic round the world: a review. Talanta. 2002;58(1):201-35.

2. Vahter M.Health effects of early life exposure to arsenic. Basic Clin Pharmacol Toxicol. 2008;102(2):204-11. doi: 10.1111/j.17427843.2007.00168.x.

3. Agencia para Sustancias Tóxicas y el Registro de Enfermedades. (ATSDR). 2007. Reseña Toxicológica del Arsénico (versión actualizada) (en inglés). Atlanta, GA: Departamento de Salud y Servicios Humanos de EE. UU., Servicio de Salud Pública. [citado diciembre de 2016]. Disponible en: https://www.atsdr.cdc.gov/ es/toxfaqs/es_tfacts $2 . h t m l$

4. Ministerio de Salud. Análisis de la Situación de Salud de Tacna 2014. Tacna: Dirección Regional de Salud, Dirección Ejecutiva de Epidemiología; 2014. Disponible en: https://es.scribd.com/doc/284314070/ Asis-tacna-v02-2014

5. Organización Mundial de la Salud. [Internet]. Ginebra: OMS; 2016 [citado diciembre de 2016. Disponible en: http:// www.who.int/mediacentre.

6. Ministerio de Salud. Reglamento de la calidad de Agua para Consumo Humano D.S. $N^{\circ}$ 031-2010-SA. Lima: Dirección General de Salud Ambiental, Ministerio de Salud; 2011. Disponible en: http:// www.digesa.minsa.gob.pe/publicaciones/ descargas/Reglamento_Calidad_Agua.pdf

7. Dirección Regional de Salud. Programa de vigilancia de la calidad del agua para consumo humano. Calidad del agua para consumo humano region Tacna. Tacna: Dirección Regional de Salud, Dirección ejecutiva de salud ambiental; 2013.

8. Ministerio de Salud. Guía de práctica clínica para el diagnóstico y tratamiento de la intoxicación por arsénico. Lima: Dirección General de Salud de las Personas, Ministerio de Salud; 2012. p. 3-19. Disponible en: http:// bvs.minsa.gob.pe/local/minsa/2109.pdf

9. Chen Y, Karagas MR. Arsenic and cardiovascular disease: new evidence from the United States. Ann Intern Med. 2013;159(10):713-4.

10. Medrano MA, Boix R, Pastor-Barriuso R, Palau M, Damian J, Ramis R, et al. Arsenic in public water supplies and cardiovascular mortality in Spain. Environ Res. 2010;110(5):448-54. doi: 10.1016/j.envres.2009.10.002.

11. Agency for Toxic Substances and Disease Registry (ATSDR). Atlanta, GA: ATSDR; 2018. Available from: https:// www.atsdr.cdc.gov/

12. Ministerio de Salud. Hidroarsenicismo Crónico Regional Endémico HACRE: 
Módulo de Capacitación. Buenos Aires: Programa Nacional de Prevención y Control de las Intoxicaciones, Ministerio de Salud de la Nación; 2011. Disponible en: http://www.msal.gob.ar/images/stories/ bes/graficos/0000000332cnt-03-Capacit_ hidroarsenicismo.pdf

13. Ministerio de Salud. Análisis de la Situación de Salud de Tacna 2017. Tacna: Dirección Regional de Salud, Dirección Ejecutiva de Epidemiología; 2014. Disponible en: https://es.scribd.com/document/364805494/Asis-Tacna-2017

14. Dirección Ejecutiva de Epidemiología. Población total por microredes. Tacna: Dirección Ejecutiva de Planeamiento Estratégico, Dirección Regional de Salud; 2016.

15. Gaioli M, González DE, Amoedo D. Hidroarcenisismo crónico regional endémico: un desafío diagnóstico y de prevención. Arch argent pediatr. 2009;107(5):459-73.

16. Chávez Reuiz M. Colección, conservación, almacenamiento, transporte y recepción de muestras biológicas para la determinación de metales pesados. Lima: Instituto Nacional de Salud, Centro Nacional de Salud
Ocupacional y protección del ambiente para la Salud; 2014.

17. Chakraborti D, Rahman MM, Ahamed S, Dutta RN, Pati S, Mukherjee SC. Arsenic groundwater contamination and its health effects in Patna district (capital of Bihar) in the middle Ganga plain, India. Chemosphere. 2016;152:520-9. doi: 10.1016/j.chemosphere.2016.02.119.

18. Smith AH, Lopipero PA, Bates MN, Steinmaus CM. Arsenic epidemiology and drinking water standards. Science. 2002;296(5576):2145-6.

19. Hurtado Y. Determinación de la exposición crónica a arsénico por consumo de agua en pobladores de dos distritos de la región Tacna, Perú. Lima: Instituto Nacional de Salud, Centro Nacional de Salud Ocupacional y protección del ambiente para la Salud; 2014.

20. Pérez Vásquez PA. Evaluación de Arsénico en orina de pobladores adultos del distrito de Ite. Tacna 2012 [Tesis]. Tacna: Universidad Nacional Jorge Basadre Grohmann; 2012. Disponible en: http://repositorio. unjbg.edu.pe/bitstream/handle/UNJBG/2399/125_2013_perez_vasquez_ pa_facs_farmacia.pdf ?sequence $=1$ \&isAllowed =y

21. Abernathy CO, Thomasy DJ, Calderon RL. Health effects and risk assessment of arsenic. J Nutr. 2003;133(5 Suppl 1):1536S-8S. doi: $10.1093 / \mathrm{jn} / 133.5 .1536 \mathrm{~S}$.

22. Rossman T. Environmental and Occupational Medicine. 4th ed. Hagerstown, MD: Lippincott Williams \& Wilkins; 2010.

23. Peña CE, Carter DE, Ayala-Fierro F. Toxicologia Ambiental: Evaluación de riesgos y restauración ambiental. Arizona: Universidad de Arizona; 2001. Disponible en: http://www.ingenieroambiental.com/ informes2/toxamb.pdf

24. Freeman K. Nutrientes protectores contra la toxicidad del arsénico: el folato y la cisteína apoyan la metilación en los niños. Salud pública Méx. 2009; 51(4):354-5.

Correspondencia: Diego André Ale Mauricio Dirección: Pj. Leoncio Prado Calle 07 de Junio 1015 B. Tacna. Perú.

Teléfono: (+51) 954478612

Correo electrónico:diegoa.mauricio@gmail.com 